fighting of some kind ensues with resulting average selective disabilities, $D_{1}$ to the victor (which gets the food) and $D_{2}$ to the loser; presumably $D_{1}$ is less than $D_{2}$.

Now let there exist a partly heritable timidity such that individuals possessing it retreat whenever another individual attacks them. They receive none of the contested food but also negligible selective disability from fighting. Clearly it will be to the advantage of an individual that would lose all its fights, if it could retreat without fighting. However, even for an individual which would win all its fights there will be an advantage in retreating whenever $D_{1}$ is greater than the probability of not finding food (before starvation, or death induced indirectly by hunger) without the presence of a competitor.

In such circumstances the genotypes for cowardice will increase in frequency for a time. The aggressive individuals, however, will each have a greater advantage as their frequency diminishes, since they will have a progressively greater proportion of encounters with retreaters and therefore gain food immediately without selective disability. An equilibrium dependent on gene frequenoy will therefore be the result unless the circumstances change. It would be of interest to know if there are any examples of such an equilibrium at present.

Further or alternative evolution is easily possible. Warning behaviour can be selected for directly as a result of use by retreaters against other retreaters. Peck orders can be established by retreat only when the opponent is known to be a better fighter. Aggression only or mainly when another individual is met in a certain territory, and retreat by the individual warned or attacked, will lead to territoriality.

Nevertheless, the mechanism proposed here does not seem adequate for explaining the evolution of the stress syndrome (if, as seems probable, this exists) or the common phenomenon of fertility reduction in relatively dense populations, except in cases of parental care. Interpopulational selection remains the only known mechanism for their origin.

Lack $^{2}$ has presented a generally similar idea; but apparently uses it only to explain the plausibility of maintenance of territoriality (in birds), since no mention is made of even phenotypic differences between individual birds in their reactions. As Lack indicates, the same bird might occupy a site or not depending on whether it was already occupied.

I thank Mr. J. Maynard Smith and Dr. D. Lack for helpful suggestions. This work was supported by a Nato post-doctoral followship.

Department of Zoology,

University College, London.

- Wynne-Edwards, V. C., Animal Dispersion in Relation to Social Behaviour (Oliver and Boyd, Edinburgh, 1962).

' Lack, D., The Natural Regulation of Animal Numbers, 270 (Clarendon Press, Oxford, 1954).

\section{Ovulation, Implantation and Fœtal Sex Ratio in the Uganda Kob}

IN twelve pregnant females of the Uganda kob (Adenota $k o b$ ) all reproductive tracts of which were examined, the single young was present in the right uterine horn regardless of whether the ovulation had occurred from the left or right ovary. In this respect the Uganda kob is like the impala (Aepyceros melampus) ${ }^{1}$.

Five virgin uteri had right uterine horns which were larger or longer than the left horns. Two parous nonpregnant uteri showed their right horns stretched more than the left. As evidenced by gross examination for corpora lutea, the ovulation occurred from both ovaries. Five recent ovulations from the right ovary and six from the left could be stated. No signs of embryo resorption or wastage of the oocytes were found. Five males and seven females were observed among the embryos, which were old enough for macroscopical determination of the sex. This result is not significantly different from a $1: 1$ sex ratio. As these results are in good agreement with those obtained in the impala ${ }^{\mathrm{I}}$ a more general significance should indubitably be attached to them.

Department of Histology,

Free University, Amsterdam.

${ }^{1}$ Mossman, A. S., and Mossman, H. W., Science, 137, 869 (1962).

\section{Prolongation of Homograft Survival in Guinea Pigs treated with Amethopterin}

AMETHOPTERIN inhibits the production of humoral antibody ${ }^{1}$ and the homograft response ${ }^{2}$ in the mouse. In view of the finding by Friedman et al. ${ }^{3}$ that amethopterin suppresses the production of humoral antibody in the guinea pig, the effect of this substance on the homograft reaction in this species was investigated.

Outbred, white-haired, male guinea pigs, weighing 250-600 $\mathrm{g}$ at the time of grafting, were fed on BruceParkes diet No. 18, supplemented with cabbage and vitamin C. Full-thickness grafts of abdominal skin, 1-2 $\mathrm{cm}^{2}$ in area, were exchanged between pairs of animals, being fitted to suprapannicular beds on the chest wall. Grafts were first inspected on the 10th day and usually every 1-2 days thereafter. They were classified as 'intact' (resembling autografts in consistency, vascularity and thickness, or with no more than a faint pinkness), "partly rejected' (firm, and a definite pink, red or purple and/or thick and/or contracted, but with a persisting circulation) or 'rejected' (yellow, wet and necrotic or dry and brown or black, and with no evidence of a vascular supply).

Amethopterin ('Methotrexate', Lederle Laboratories) was suspended in physiological saline and injected intraperitoneally on Mondays, Wednesdays and Fridays of each week, beginning on the day of grafting (Mondays) and continuing until rejection of the graft or death of the animal. The doses used were 9,12 or $15 \mathrm{mg} / \mathrm{kg}$ per injection.

Ninety-eight animals were used. Those dying before the first inspection were excluded from the series; they comprised $3 / 44$ uninjected controls and $1 / 16,2 / 21$ and $1 / 17$ in the three amethopterin-treated groups. The results are summarized in Fig. 1.

About 30 per cent of the control grafts were intact at first inspection, compared with 60,90 and 94 per cent in the three groups on amethopterin. Only about 5 per cent of control grafts survived more than 3 weeks, compared with 27,63 and 44 per cent in the amethopterin-treated groups. For estimation of graft mean survival times it was assumed that: (a) grafts rejected at first inspection had been rejected on day 9; $(b)$ grafts persisting until death of the recipient would not have survived longer had the recipient lived; (c) grafts persisting at the end of the experiment would not have survived longer had the period of observation been extended. The effect of these assumptions was to minimize the differences between groups. The mean survival times of grafts in the control group was 12.7 days and, in the groups on 9,12 and $15 \mathrm{mg} / \mathrm{kg}$ amethopterin, $24 \cdot 1,35 \cdot 8$ and $22 \cdot 4$ days respectively. The distribution of recorded survival times made estimation of standard deviations inappropriate. Instead, $2 \times 2$ contingency tables were constructed comparing the incidences of surviving and rejected grafts in pairs of groups at 4-day intervals. The results are given in Table 1.

Table 1. Probabmimtes (Exact METHOD, ONR-TAMED TEST) THAT THE LONGER SURVIVAL TIMES OF GRAFTS IN THE SECOND AS COMPARED WITH

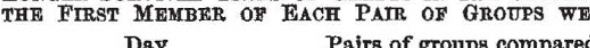

\begin{tabular}{cccc} 
Day & \multicolumn{3}{c}{ Pairs of groups compared } \\
& Control-9 mg & $9-12 \mathrm{mg}$ & $15-12 \mathrm{mg}$ \\
$11 \frac{1}{2}$ & 0.15 & 0.3 & 0.3 \\
$15 \frac{1}{2}$ & 0.02 & 0.1 & 0.3 \\
$19 \frac{1}{2}$ & 0.03 & 0.04 & 0.2 \\
$23 \frac{1}{2}$ & 0.03 & 0.04 & 0.06 \\
$27 \frac{1}{2}$ & 0.06 & 0.25 & 0.5
\end{tabular}

